\title{
Corrigendum: T cell killing does not require the formation of a stable mature immunological synapse
}

\author{
Marco A Purbhoo, Darrell J Irvine, Johannes B Huppa \& Mark M Davis \\ Nature Immunology 5, 524-530 (2004).
}

In the legend to Figure 4, the last sentence should be replaced with the following: "Curves in $\mathbf{b}$ and $\mathbf{d}$ represent the average of at least eight CTLAPC conjugates each.” The authors also regret having omitted reference to M. Faroudi et al. Lytic versus stimulatory synapse in cytotoxic T lymphocyte/target cell interaction: manifestation of a dual activation threshold. Proc. Natl. Acad. Sci. USA 100, 14145-14150 (2003), which showed that with a human cytotoxic T lymphocyte line, lower concentrations of the antigenic peptide than were needed for mature synapse formation could nonetheless induce cytotoxicity. This should be cited on page 528, column 2, at the end of the first paragraph.

\section{Corrigendum: Selective associations with signaling proteins determine stimulatory versus costimulatory activity of NKG2D}

\author{
Andreas Diefenbach, Elena Tomasello, Mathias Lucas, Amanda M Jamieson, Jennifer K Hsia, Eric Vivier \& David H Raulet \\ Nature Immunology 3, 1142-1149 (2002).
}

\begin{abstract}
Although the major conclusions of this paper, that the NKG2D immunoreceptor in mice exists in two splice isoforms that associate differentially with DAP10 and DAP12 and contribute to distinct signaling properties of the receptor in CD8 T cells as opposed to NK cells, are not affected, the authors wish to alert the community that recent experiments with macrophages do not bear out our conclusion that macrophages express easily detectable amounts of NKG2D on the cell surface. In recent repetitions of the staining experiments, we have failed to obtain clear-cut staining of activated bone marrow or peritoneal macrophages with antibodies to NKG2D. Similarly, efforts to elicit a functional response from activated macrophages by cross-linking NKG2D with antibodies to NKG2D yielded either no response or a questionable response. Low expression is still possible, because we confirmed earlier studies that activated macrophages do contain detectable mRNA for NKG2D, and it is known that these cells express the necessary adapter proteins for surface expression of the receptor.

The new data also bear on one conclusion of an earlier paper from Diefenbach, A., Jamieson, A.M., Liu, S.D., Shastri, N. \& Raulet, D.H. Ligands for the murine NKG2D receptor: expression by tumor cells and activation of NK cells and macrophages. Nature Immunology $1,119-126$ (2000). The conclusion in that paper that activated peritoneal macrophages express readily detectable NKG2D at the cell surface is now also in question, but the conclusions concerning natural killer cells and CD8 T cells remain unchanged.
\end{abstract}

